\title{
A Weberized Total Variation Regularization-Based Image Multiplicative Noise Removal Algorithm
}

\author{
Liang Xiao, Li-Li Huang, and Zhi-Hui Wei \\ 603 LAB, School of Computer Science and Technology, Nanjing University of Science and Technology, Nanjing 210094, China
}

Correspondence should be addressed to Liang Xiao, xiaoliang@mail.njust.edu.cn

Received 29 April 2009; Revised 5 October 2009; Accepted 16 February 2010

Academic Editor: Yasar Becerikli

Copyright () 2010 Liang Xiao et al. This is an open access article distributed under the Creative Commons Attribution License, which permits unrestricted use, distribution, and reproduction in any medium, provided the original work is properly cited.

Multiplicative noise removal is of momentous significance in coherent imaging systems and various image processing applications. This paper proposes a new nonconvex variational model for multiplicative noise removal under the Weberized total variation (TV) regularization framework. Then, we propose and investigate another surrogate strictly convex objective function for Weberized TV regularization-based multiplicative noise removal model. Finally, we propose and design a novel way of fast alternating optimizing algorithm which contains three subminimizing parts and each of them permits a closed-form solution. Our experimental results show that our algorithm is effective and efficient to filter out multiplicative noise while well preserving the feature details.

\section{Introduction}

Image denoising is one of the fundamental problems in image processing and computer vision. Multiplicative noise appears in various image processing applications, for example, in synthetic aperture radar (SAR), ultrasound imaging, single particle emission computed tomography (SPECT), and positron emission tomography (PET) [1]. Hence, multiplicative noise removal is of momentous significance in coherent imaging systems and various image processing applications.

The essential idea for image denoising is to filter out noise in an image without losing significant features such as edges and textures. However, one of the challenges in image denoising is its ill-posed nature. To cope with this problem, a large number of approaches have been proposed, most of them under the regularization or the Bayesian frameworks $[2,3]$. These approaches are supported on some form of a priori knowledge or regularization about the original image to be estimated. Some of these methods, including Markov random field priors, wavelet-based priors or regularization $[4,5]$, curvelet-based diffusion [6], and total variation (TV) regularization [7-9] are considered the state-of-the-art. Among these approaches, variational functional regularization and partial differential equations(PDE-) based models have become international popular issues. These models provide a good theoretical foundation to the image denoising task and other inverse problems such as image segmentation and image inpainting, and so forth. And they also simulate the human vision well because of its close relationship to multi-scale analysis theory [10].

1.1. Background. Although other types of noise (e.g., impulse or Poisson noise) have also been studied in the literature of image processing, the term "image denoising" is usually devoted to the problem associated with additive Gaussian noise. The goal of image denoising is to recover ideal $u$ from the observed noisy data $u_{0}=u+v$, where $u_{0}$ is an observed image defined on $\Omega$, and $\Omega \subset R^{2}$ denotes an open bounded set with Lipschitz boundary, and $v$ denotes the additive Gaussian noise. To cope with the ill-posed nature of denoising, the regularization techniques are often utilized to solve such a problem. Specifically, the regularization functional-based denoising is given by

$$
\widehat{u}=\arg \min _{u}\left\{E_{\text {smooth }}(u)+\lambda E_{\text {data }}\left(u, u_{0}\right)\right\},
$$

where $E_{\text {data }}\left(u, u_{0}\right)$ is the image fidelity term depending upon the additive noise model, which penalizes the inconsistency between the underestimated recovery image and the acquired noisy image, $E_{\text {smooth }}(u)$ is the regularization term which imposes some priori constraints on the original image and 
to a great degree determines the quality of the recovery image, and $\lambda$ is the regularization parameter which controls the trade-off between the image fidelity term $E_{\text {data }}\left(u, u_{0}\right)$ and the regularization term $E_{\text {smooth }}(u)[2,3,9,11]$. The classical model is the minimizing total variational functional [7] $E_{\text {smooth }}(u)=\operatorname{TV}(u)=\int_{\Omega}|\nabla u| \mathrm{d} x$ with least square data fidelity $E_{\text {data }}\left(u, u_{0}\right)=\int_{\Omega}\left(u-u_{0}\right)^{2} \mathrm{~d} x$. We refer the readers to $[2,3]$ and references herein for an overview of the subject [9].

In this paper, we focus on the issue of multiplicative noise removal. Specifically, we are interested in the denoising of SAR images. According to [12] and other references, the noise in the observed SAR image is a type of multiplicative noise which is called speckle. And the image formation model is

$$
u_{0}=u v
$$

where $u_{0}$ is the observed image, $u$ is the original SAR image, and $v$ is the noise which follows a Gamma Law with mean one with its probability density function given by

$$
f_{V}(v)=\frac{L^{L}}{\Gamma(L)} v^{L-1} e^{(-L v)} \cdot 1\{v>0\},
$$

where $L$ is the number of looks (in general, an integer coefficient) and $\Gamma(\cdot)$ is a Gamma function. Speckle is one of the more complex image noise models. It is signal independent, non-Gaussian, and spatially dependent. Hence, speckle denoising is a very challenging problem compared with additive Gaussian noise.

1.2. Prior Works on Variational Approaches. Multiplicative noise removal methods have been discussed in many reports. Popular methods include the Lee method [13], the Kuan method [14], and various anisotropic diffusion-based methods [15-17], which will not be addressed in this paper. We will focus on the variational approaches-based multiplicative noise removal, especially that our researches will emphasis on the TV model-based methods.

To the best of our knowledge, there exist only several variational approaches devoted to multiplicative noise removal problem. The first total variation-based multiplicative noise removal model was presented by Rudin et al. [18], which used a constrained optimization approach with two Lagrange multipliers. The authors derived a denoising model (RLO) as follows:

$$
\begin{array}{ll}
\min & \int_{\Omega}|\nabla u| \mathrm{d} x \\
\text { s.t. } & \left\{\begin{array}{l}
\int_{\Omega} v \mathrm{~d} x=1, \\
\int_{\Omega}(v-1)^{2} \mathrm{~d} x=\sigma^{2}
\end{array}\right.
\end{array}
$$

and designed a gradient projection-based algorithm. Following the maximum a posteriori (MAP) estimator for multiplicative Gamma noise, Aubert and Aujol [19] introduced a nonconvex model (AA):

$$
\min _{u}\left\{E(u)=\operatorname{TV}(u)+\lambda \int_{\Omega}\left(\log u+\frac{u_{0}}{u}\right) \mathrm{d} x\right\},
$$

where $E_{\text {data }}\left(u, u_{0}\right)=\int_{\Omega}\left(\log u+u_{0} / u\right) \mathrm{d} x$ is the data fitting term and $E_{\text {smooth }}(u)=\operatorname{TV}(u)=\int_{\Omega}|\nabla u| \mathrm{d} x$ is the total variation (TV) regularization term. They also used gradient descend method to solve AA model. Recently, Shi and Osher [20] adopted a noisy observation $\log u_{0}=\log u+$ $\log v$ and the data term of the AA model then derived the TV minimization model for multiplicative noise removal problems. They applied a corresponding relaxed inverse scale space flow as denoising technique. Huang et al. [21] proposed a strictly convex model by letting $w=$ $\log u$ and replacing $\operatorname{TV}(w)$ by $\operatorname{TV}(u)$ based on the prior work in [19], and they proposed a simpler alternating minimization algorithm by adding a quadratic term to the model. A variational model involving curvelet coefficients for cleaning multiplicative Gamma noise was considered in [22]. Steidl and Teuber [1] introduced a variational restoration model consisting of the I-divergence as data fitting term and the total variation seminorm as regularizer. They applied Douglas-Rachford splitting techniques, respectively, alternating split Bregman methods to denoising.

1.3. Contributions. The main aim of this paper is to propose and investigate another nonconvex variational model for multiplicative noise removal which inspiring from the Weberized TV regularization method [23, 24]. We also incorporate another way of surrogate functional model to recover image edges. We develop an alternating minimization algorithm to find the minimizer of such an objective function efficiently. Our experimental results show that our proposed method has good performance for multiplicative noise removal. The outline of this paper is as follows. In Section 2, we introduce our Weberized total variation-based multiplicative noise removal model. In Section 3, we propose a surrogate functional model which can be viewed as an approximating way of the new nonconvex model. In Section 4, we develop a novel fast algorithm for minimizing such surrogate functional model. In Section 5, we show experimental results to demonstrate the quality of the denoised images and the efficiency of our proposed algorithm. Finally, concluding remarks are given in Section 6 .

\section{Weberized TV Regularization Model for Multiplicative Noise Removal}

All images are eventually perceived and interpreted by the Human Visual System (HVS). As a result, many researchers have found that human vision psychology and psychophysics play an important role in image processing. The classical example is the using of the Just Noticeable Difference Model (JND) in image coding and watermarking techniques [25, 26]. In these fields, the JND model is used to control the visual perceptual distortion during the coding procedure and watermark embedding. Weber's law was first described in 1834 by German physiologist Weber [27]. The law reveals the universal influence of the background stimulus $u$ on human's sensitivity to the intensity increment $|\nabla u|$, 
or so-called JND, in the perception of both sound and light:

$$
\frac{|\nabla u|}{u}=\text { const. }
$$

According to Weber's law, when the mean intensity of the background is increasing with a higher value, the intensity increment $|\nabla u|$ also has higher value. In literature [23], the author gave a complete analysis and report for the Gaussian denoising problem:

$$
u_{0}=u+v .
$$

He proposed a nonconvex variational model:

$$
\min _{u}\left\{E(u)=\operatorname{TV}(\log u)+\lambda\left\|u_{0}-u\right\|_{L^{2}(\Omega)}\right\} .
$$

The essential idea of the above model is the use of Weber's law. For the optimizing model (8), the author had given complete existence and uniqueness theorem in the following natural admissible space $S(\Omega)$ defined as

$$
S(\Omega)=\left\{u>0\left|u \in L^{2}(\Omega), \int_{\Omega}\right| \nabla \log u \mid \mathrm{d} x<\infty, u>\frac{u_{0}}{2}\right\}
$$

and presented a fast gradient descent algorithm.

Inspired from the Weberized TV regularization method, we propose a nonconvex Weberized TV regularization-based multiplicative noise removal model:

$$
\begin{aligned}
\widehat{u} & =\arg \min _{u}\left\{E(u)=\operatorname{TV}(\log u)+\lambda \int_{\Omega}\left(\frac{u_{0}}{u}+\log u\right) \mathrm{d} x\right\} \\
& =\arg \min _{u}\left\{E(u)=\int_{\Omega} \frac{|\nabla u|}{u} \mathrm{~d} x+\lambda \int_{\Omega}\left(\frac{u_{0}}{u}+\log u\right) \mathrm{d} x\right\},
\end{aligned}
$$

where the first term $E_{w}(u):=\operatorname{TV}(\log u)=\int_{\Omega}(|\nabla u| / u) \mathrm{d} x$ is the well-known Weberized TV regularization term, while the second one is the nonconvex data fidelity term. Comparing the model in (10) with the model in (5), it is very interesting to see that the model in (10) is essentially similar to Weberized TV Regularization for Gaussian noise removal model (8), and the difference is that the data fidelity term is adjusted by AA model's data fidelity term. Furthermore, the proposed model (10) can be understood and interpreted from the statistical perspective using Bayesian formulation, and this is straightforward application of MAP theory as illustrated in $[19,21]$.

In addition, one may wonder whether the minimizing problem of (10) has the unique solution or not. It is very important to point that existence and uniqueness of this problem can also be valid and can be proved. The proof can be made very similar to the discussion for the model (8), which can be found in [13].

\section{The Proposed Surrogate Functional Model and Mathematical Analysis}

3.1. The Proposed Surrogate Functional Model. In this subsection, we will propose another extension strictly convex objective function which can be viewed as a surrogate functional model for minimizing Weberized TV regularization problems (10). To do this, we first give the formal equilibrium Euler-Lagrange equation of problem (10). For the sake of convenience, let us denote that $\Phi(u)=1 / u$.

Lemma 1. Let $\Phi(u): R^{+} \rightarrow R^{+}$be a $C^{1}$ function and

$$
E(u)=\int_{\Omega} \Phi(u)|\nabla u| d x+\lambda \int_{\Omega}\left(\frac{u_{0}}{u}+\log u\right) d x,
$$

then the formal equilibrium Euler-Lagrange equation of $E(u)$ is

$$
\begin{gathered}
-\Phi(u) \operatorname{div}\left(\frac{\nabla u}{|\nabla u|}\right)+\lambda \frac{u-u_{0}}{u^{2}}=0, \\
\left.\frac{\partial u}{\partial \vec{n}}\right|_{\partial \Omega}=0,
\end{gathered}
$$

where $\vec{n}$ is the outward normal to $\partial \Omega$ and $\vec{n}=(\nabla u /|\nabla u|)$.

Proof. Note that in the first integral of the second line, by the standard computation of the operator $\operatorname{div}(\Phi(u) \nabla u /|\nabla u|)$, it is easy to prove that

$$
\operatorname{div}\left(\Phi(u) \frac{\nabla u}{|\nabla u|}\right)=\Phi(u) \cdot \operatorname{div}\left(\frac{\nabla u}{|\nabla u|}\right)+\Phi^{\prime}(u)|\nabla u| .
$$

Then we use the standard computation of Calculus of Variation $E \rightarrow E+\delta E$ :

$$
\begin{aligned}
\delta E= & \int_{\Omega}\left(\Phi^{\prime}(u)|\nabla u| \delta u+\Phi(u) \frac{\nabla u}{|\nabla u|} \nabla(\delta u)\right) \mathrm{d} x \\
& +\lambda \int_{\Omega} \frac{u-u_{0}}{u^{2}} \delta u \mathrm{~d} x \\
= & \int_{\Omega}\left(\Phi^{\prime}(u)|\nabla u|-\operatorname{div}\left(\Phi(u) \frac{\nabla u}{|\nabla u|}\right) \delta u\right) \mathrm{d} x \\
& +\int_{\partial \Omega} \frac{\Phi(u)}{|\nabla u|} \frac{\partial u}{\partial \vec{n}} \delta u \mathrm{~d} s+\lambda \int_{\Omega} \frac{u-u_{0}}{u^{2}} \delta u \mathrm{~d} x \\
= & \int_{\Omega}\left(-\Phi(u) \cdot \operatorname{div}\left(\frac{\nabla u}{|\nabla u|}\right)\right) \delta u \mathrm{~d} x \\
& +\int_{\partial \Omega} \frac{\Phi(u)}{|\nabla u|} \frac{\partial u}{\partial \vec{n}} \delta u \mathrm{~d} s+\lambda \int_{\Omega} \frac{u-u_{0}}{u^{2}} \delta u \mathrm{~d} x,
\end{aligned}
$$

where $\mathrm{d} s$ denotes the arc-length element of the boundary. This completes the proof.

Since $u>0, \Phi(u)=1 / u$, then the Euler-Lagrange equation (12) can be rewritten equivalently as

$$
\begin{gathered}
-\operatorname{div}\left(\frac{\nabla u}{|\nabla u|}\right)+\lambda \frac{u-u_{0}}{u}=0, \\
\left.\frac{\partial u}{\partial \vec{n}}\right|_{\partial \Omega}=0 .
\end{gathered}
$$


Suppose that there exists a function $M(u)$ which satisfies $M^{\prime}(u)=\left(u-u_{0}\right) / u$, then it is easy to find the function $M(u)=u-u_{0} \log u$, and then (15) is also the corresponding Euler-Lagrange equation of another objective functional or a new reference energy $E_{R}(u)$ which is defined as follows:

$$
E_{R}(u)=\int_{\Omega}|\nabla u| \mathrm{d} x+\lambda \int_{\Omega}\left(u-u_{0} \log u\right) \mathrm{d} x .
$$

Note that optimization problems given in (10) and (16) have the same equilibrium Euler-Lagrange equation (15). In Section 3.2, we can prove that the solution of optimizing model (16) admits a unique solution. In other words, on one hand, the unique solution of optimization problems given in (10) is also a solution of optimizing model (16). On the other hand, optimizing model (16) admits a unique solution which satisfies (15). Taking into consideration two facts mentioned above, it is clear to see that the equivalence of optimization problems given in (10) and (16) can be guaranteed. Hence, model (16) can be viewed as a surrogate functional for minimizing the Weberized TV regularization problem (10).

3.2. Existence and Uniqueness Results. As discussed in Section 3.1, to show the equivalence of optimization problems given in (10) and (16), we need to prove the existence and uniqueness of solution in optimizing model (16). To do this, we briefly recall some notations and basic preliminaries of $\operatorname{BV}(\Omega)$ (see $[3,24])$. Let $C_{0}^{p}(\Omega)$ denote the space of real-valued functions, $p$ is continuously differentiable with compact support, let $L^{p}(\Omega)$ denote the space of Lebesgue measurable functions $u$ such that $\int_{\Omega}|u|^{p} \mathrm{~d} x<\infty$ and $L^{\infty}(\Omega)$ the space of Lebesgue measurable functions $u$ such that there exists a constant $c$ with $|u(x)| \leq c$ a.e. $x \in \Omega$.

Definition 1. Let $\mathrm{BV}(\Omega)$ be a space of function of $u \in L^{1}(\Omega)$ such that the following quantity:

$\int_{\Omega}|\nabla u|=\sup \left\{\int_{\Omega} u \operatorname{div}(\vec{g}) \mathrm{d} x\left|\vec{g} \in C_{0}^{1}\left(\Omega ; R^{2}\right),\right| \vec{g} \mid \leq 1\right\}$

is finite. $\mathrm{BV}(\Omega)$ is a Banach space with the norm $\|u\|_{\mathrm{BV}(\Omega)}=$ $\mathrm{TV}(u)+\|u\|_{L^{1}(\Omega)}$.

We summarize below the lower semicontinuity and compactness properties of $\operatorname{BV}(\Omega)[3,28]$ that we will use in the proof.

(i) Suppose that $u_{k} \in \operatorname{BV}(\Omega)(k=1,2, \ldots)$ and $u_{k} \rightarrow u$ in $L_{\text {loc }}^{1}(\Omega)$, then $\int_{\Omega}|\nabla u| \mathrm{d} x \leq$ $\lim _{n \rightarrow \infty} \inf \int_{\Omega}\left|\nabla u_{n}\right| \mathrm{d} x$.

(ii) Suppose that $\left\{u_{n}\right\}_{n=1}^{\infty}$ is a sequence in $\operatorname{BV}(\Omega)$ satisfying $\sup _{n}\left\|u_{n}\right\|_{\mathrm{BV}(\Omega)}<\infty$, then there exists a subsequence $\left\{u_{n_{j}}\right\}_{j=1}^{\infty}$ and a function $u \in \operatorname{BV}(\Omega)$ such that $u_{n_{j}} \rightarrow u$ in $L^{1}(\Omega)$ as $j \rightarrow \infty$.

Using the lower semicontinuity and compactness of $\mathrm{BV}(\Omega)$, we can conclude the following existence and uniqueness theorem.
Theorem 1. Let $u_{0} \in L^{\infty}(\Omega)$ be a positive, bounded function with $\inf _{\Omega} u_{0}>0$, then the minimizing problem of energy functional in (16) admits a unique solution $u \in \operatorname{BV}(\Omega)$ satisfying

$$
\inf _{\Omega}\left(u_{0}\right) \leq u \leq \sup _{\Omega}\left(u_{0}\right)
$$

Proof. See the appendix.

\section{Proposed Fast Algorithm}

4.1. Motivation. In this subsection, we will develop a fast multiplicative noise removal algorithm for the optimizing energy functional of (16). Our algorithm is designed using the well-known variable-splitting and penalty techniques in optimization [20-22]. An auxiliary variable $w$ is firstly introduced to transfer $u$ out of the nondifferentiable term $\operatorname{TV}(u)$, and the difference between $w$ and $u$ is penalized, yielding the following approximation model of model (16):

$$
\begin{aligned}
\min _{u, w}\left\{E_{s}(u, w)=\right. & \int_{\Omega}|\nabla w| \mathrm{d} x+\alpha_{2} \int_{\Omega}|w-u|^{2} \mathrm{~d} x \\
& \left.+\alpha_{3} \int_{\Omega}\left(u-u_{0} \log u\right) \mathrm{d} x\right\},
\end{aligned}
$$

with a sufficiently large penalty parameter $\alpha_{2}>0$. It is well known that the solution of (19) converges to that of (16) as $\alpha_{2} \rightarrow \infty$. While either one of the two variables $u$ and $w$ is fixed, problem (19) can be solved by alternative iterative subproblem minimizing:

(i) $\hat{u}=\arg \min _{u}\left\{\alpha_{2} \int_{\Omega}|w-u|^{2} \mathrm{~d} x\right.$

$$
\left.+\alpha_{3} \int_{\Omega}\left(u-u_{0} \log u\right) \mathrm{d} x\right\}
$$

(ii) $\widehat{w}=\arg \min _{w}\left\{\int_{\Omega}|\nabla w| \mathrm{d} x+\alpha_{2} \int_{\Omega}|w-u|^{2} \mathrm{~d} x\right\}$.

The main advantage of this procedure is that the proposed model takes advantage of using the total variation minimization scheme to remove the multiplicative noise. It can be solved by many TV denoising fast methods such as the Chambolle projection algorithm [29], primal-dual method, and the lagged diffusivity fixed point method [30-33]. In this paper, we will use another fast algorithm proposed in $[34,35]$ to deal with TV denoising problem (21). Similar to the previous discussion, we introduce another vector variable $v=\left(v_{1}, v_{2}\right)$ to approximate $\nabla w$, and then subproblem $(21)$ is approximated by

$$
\begin{gathered}
(\hat{v}, \widehat{w})=\arg \min _{v, w}\left\{\int_{\Omega}|v| \mathrm{d} x+\alpha_{1} \int_{\Omega}|\nabla w-v|^{2} \mathrm{~d} x\right. \\
\left.+\alpha_{2} \int_{\Omega}|w-u|^{2} \mathrm{~d} x\right\},
\end{gathered}
$$

where $\alpha_{1}>0$ is the penalty parameter, and the solution of (22) converges to that of (21) as $\alpha_{1} \rightarrow \infty$. 
4.2. Discrete Scheme. Let us consider the discrete scheme of the problem. Let matrix $u_{0} \in R^{N \times N}$ represent a two dimensional gray-scale digital image, where each component $\left(u_{0}\right)_{i, j}$ is the intensity value of pixel $(i, j)$ for $i, j \in\{1,2, \ldots, N\}$. Similarly, we let matrix $u$ represent the unknown image to be restored. Let us define the forward finite difference operator

$$
(D u)_{i j}=\left(\begin{array}{c}
\left(D_{1} u\right)_{i j} \\
\left(D_{2} u\right)_{i j}
\end{array}\right)=\left(\begin{array}{c}
u_{i+1, j}-u_{i, j} \\
u_{i, j+1}-u_{i, j}
\end{array}\right), \quad 1 \leq i, j<N,
$$

with periodic boundary adjustments for $i=N$ and $j=N$, and $D$ is a block-circulant-circulant-block (BCCB) matrix. Under the above definitions, the corresponding discrete form of (20) and (22) is

(i) $u$-subproblem:

$$
\begin{aligned}
\hat{u}=\arg \min _{u}\{ & \alpha_{2} \sum_{i, j}\left\|w_{i, j}-u_{i, j}\right\|_{2}^{2} \\
& \left.+\alpha_{3} \sum_{i, j}\left(u_{i, j}-\left(u_{0} \log u\right)_{i, j}\right)\right\},
\end{aligned}
$$

(ii) $w$-subproblem:

$$
\begin{gathered}
\widehat{w}=\arg \min _{v, w}\left\{\sum_{i, j}\left\|v_{i, j}\right\|_{2}+\alpha_{1} \sum_{i, j}\left\|(D w)_{i, j}-v_{i, j}\right\|_{2}^{2}\right. \\
\left.+\alpha_{2} \sum_{i, j}\left\|w_{i, j}-u_{i, j}\right\|_{2}^{2}\right\} .
\end{gathered}
$$

4.2.1. $u$-Subproblem. Since this is strictly convex problem, it is equivalent to solve

$$
(u-w)+\frac{\alpha_{3}}{2 \alpha_{2}}\left(1-\frac{u_{0}}{u}\right)=0
$$

or (let $\gamma=\alpha_{3} /\left(2 \alpha_{2}\right)$ ) find the meaningful positive root for this equation, that is,

$$
\widehat{u}=\frac{w-\gamma+\sqrt{(w-\gamma)^{2}+4 \gamma u_{0}}}{2} .
$$

4.2.2. $w$-Subproblem. It can be solved by alternately minimizing the objective function with respect to $v$ while fixing $w$, and vice versa:

(i) $\hat{v}=\arg \min _{v}\left\{\sum_{i, j}\left\|v_{i, j}\right\|_{2}+\alpha_{1} \sum_{i, j}\left\|(D w)_{i, j}-v_{i, j}\right\|_{2}^{2}\right\}$,

(ii) $\widehat{w}=\arg \min _{w}\left\{\alpha_{1} \sum_{i, j}\left\|(D w)_{i, j}-v_{i, j}\right\|_{2}^{2}\right.$

$$
\left.+\alpha_{2} \sum_{i, j}\left\|w_{i, j}-u_{i, j}\right\|_{2}^{2}\right\} \text {. }
$$

It is not difficult to verify that the $v$-subproblem permits a closed-form solution [34]:

$$
\hat{v}_{i j}=\max \left(\left\|(D w)_{i j}\right\|_{2}-\frac{1}{2 \alpha_{1}}, 0\right) \cdot \frac{(D w)_{i j}}{\left\|(D w)_{i j}\right\|_{2}} .
$$

For a fixed $v$, let $\mu=\alpha_{1} / \alpha_{2}$, then $w$-subproblem (29) is quadratic in $w$ and the minimizer $\widehat{w}$ is given by the normal equation $[34,35]$

$$
\left[\mu\left(D_{1}^{T} D_{1}+D_{2}^{T} D_{2}\right)+I\right] w=\mu\left(D_{1}^{T} v_{1}+D_{2}^{T} v_{2}\right)+u .
$$

Under the periodic boundary condition for $w, D_{1}$, and $D_{1}$ are block circulant. So $D_{1}^{T} D_{1}$ and $D_{2}^{T} D_{2}$ are all block circulant. Therefore, the Hessian matrix on the left-hand side of (31) can be diagonalized by two-dimensional discrete Fourier transform $F$. Using the convolution theorem of Fourier transforms, we can write

$$
\widehat{w}=F^{-1}\left(\frac{F(u)+\mu \cdot \sum_{k=1}^{2} F\left(D_{k}\right)^{*} \circ F\left(v_{k}\right)}{1+\mu \cdot \sum_{k=1}^{2} F\left(D_{k}\right)^{*} \circ F\left(v_{k}\right)}\right),
$$

where the symbol “*” denotes complex conjugacy, "。” denotes component-wise multiplication, and the division is component-wise as well. With a slight abuse of notation, we have used $F\left(D_{1}\right)$ for the Fourier transform of the function represented by $D_{1}$ in the convolution $D_{1} u$ (and similarly for $\left.D_{2}\right)$. Since all quantities but $v_{1}$ and $v_{2}$ are constant, computing $\widehat{w}$ from (32) involves two FFTs and one inverse FFT, once the constant quantities are computed.

4.3. Parameters Choice. There are three parameters $\alpha_{1}, \alpha_{2}$, and $\alpha_{3}$ or equivalently $\alpha_{1}, \gamma=\alpha_{3} /\left(2 \alpha_{2}\right)$, and $\mu=\alpha_{1} / \alpha_{2}$ involved in the iterative procedure.

Firstly, from the connection between model (16) and model (19), how well the solution of (19) approximates that of (16) or its constrained equivalent depends on the magnitude of $\alpha_{1}, \alpha_{2}$, which determines the amount of penalty applied to the discrepancy between $u$ and $w$ and also between $\nabla w$ and $v$ in the squared L2-distance. Hence, the magnitude of $\alpha_{1}, \alpha_{2}$ must have larger enough value.

Secondly, $\alpha_{3}$ is the regularization parameter which controls the trade-off between the image fidelity term and the regularization term. We dynamically compute the value of $\alpha_{3}$ according to the variance of the recovered noise which matches that of our prior knowledge [7]. The Gamma distributed noise has the mean and variance as follows:

$$
\int_{\Omega} \frac{u_{0}}{u} \mathrm{~d} x=1, \quad \int_{\Omega}\left(\frac{u_{0}}{u}-1\right)^{2} \mathrm{~d} x=\sigma^{2} .
$$

Notice that the Euler-Lagrange equation of the optimizing problem of (16) is (15). The solution procedure uses a parabolic equation with time as an evolution parameter. This means that we solve

$$
\begin{gathered}
\frac{\partial u}{\partial t}=\operatorname{div}\left(\frac{\nabla u}{|\nabla u|}\right)+\alpha_{3} \frac{u_{0}-u}{u}, \\
\left.\frac{\partial u}{\partial \vec{n}}\right|_{\partial \Omega}=0,
\end{gathered}
$$


for $t>0$. We merely multiply the first equation of (34) by $\left(\left(u_{0}-u\right) / u\right)$ and integrate by parts over $\Omega$. If steady state has been reached, the left side of the first equation of (34) vanishes, then we have

$$
\alpha_{3}=\frac{1}{\sigma^{2}} \int_{\Omega}\left[\frac{\nabla u}{|\nabla u|} \cdot \nabla\left(\frac{u_{0}-u}{u}\right)\right] \mathrm{d} x
$$

4.4. Proposed Fast Algorithm. In summary, according to the iterative process analysis and parameters choice which were discussed in Sections 4.1 and 4.2, respectively, we can propose a fast algorithm framework as follows. Two loops of iterations are contained in this algorithm framework. In the outer loop, we use the continued method by increasing the parameters $\alpha_{1}$ and $\alpha_{2}$ to achieve the good convergence. At the same time, in the inner loop, we update the parameter $\alpha_{3}$ in order to match the variance of the recovered noise.

According to the optimality conditions of (20), (28), and (29), the stopping criterion of the proposed algorithm is proposed in the following. Let

$$
\begin{gathered}
r_{1}=u-w+\gamma\left(1-\frac{u_{0}}{u}\right) \\
r_{2}(i, j)=\frac{v_{i, j}}{\left(2 \alpha_{1}\left\|v_{i, j}\right\|_{2}\right)}+v_{i, j}-(D w)_{i, j}, \quad\left\|v_{i, j}\right\|_{2} \neq 0 \\
r_{3}(i, j)=2 \alpha_{1}\left\|(D w)_{i, j}\right\|_{2} \leq 1, \quad\left\|v_{i, j}\right\|_{2}=0, \\
r_{4}=\mu D^{T}(D w-v)+(w-u) .
\end{gathered}
$$

The inner loop is terminated once

$$
\begin{aligned}
& \text { Res }=\max \left\{\left\|r_{1}\right\|_{\infty}, \max _{i, j}\left\{\left\|r_{2}(i, j)\right\|_{2}\right\},\right. \\
&\left.\max _{i, j}\left\{\left\|r_{3}(i, j)\right\|_{2}\right\},\left\|r_{4}\right\|_{\infty}\right\} \leq \zeta,
\end{aligned}
$$

where Res measures the total residual and $\zeta>0$ is a prescribed tolerance.

The complete resulting algorithm is summarized in Algorithm 1.

4.5. Convergence Analysis. The convergence of the quadratic penalty method as the penalty parameter goes to infinity is well known (e.g., see [36, Theorem 17.1]). That is, as $\alpha_{1}, \alpha_{2} \rightarrow \infty$ the solution of (19) converges to that of (16), and the solution of (22) converges to that of (21), respectively. In this subsection, we present the convergence results of Algorithm 1 for fixed $\alpha_{1}$ and $\alpha_{2}$ without proofs since these results are rather straightforward application of [34, Theorem 3.4]. For the sake of completeness, we first present some necessary definitions and then give the main convergence results.

To begin with, for $a \in R^{2}$, we define the 2D shrinkage operator $s: R^{2} \rightarrow R^{2}$ by

$$
s(a)=\max \left(\|a\|_{2}-\frac{1}{2 \alpha_{1}}, 0\right) \cdot \frac{a}{\|a\|_{2}},
$$

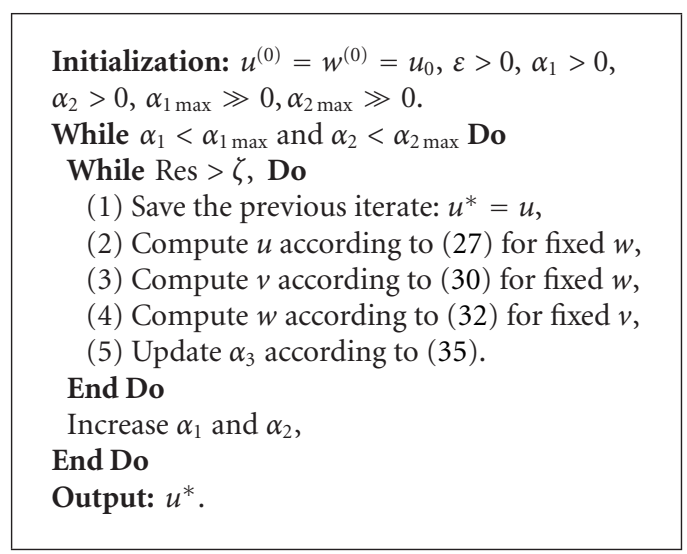

Algorithm 1

where $0 \cdot(0 / 0)=0$ is followed. An then we define an $N$ dimensional vector shrinkage operator $S(u ; v): R^{2 N^{2}} \rightarrow R^{2 N^{2}}$ by

$$
S(u ; v)=\left(s\left(u_{1}, v_{1}\right) ; \ldots ; s\left(u_{N^{2}}, v_{N^{2}}\right)\right), \quad u, v \in R^{N^{2}},
$$

that is, $S$ applies 2D shrinkage to each pair $\left(u_{i}, v_{i}\right) \in R^{2}, i=$ $1,2, \ldots, N^{2}$.

In addition, we define a linear operator $h: R^{2 N^{2}} \rightarrow R^{2 N^{2}}$ as $h(v)=\left(h_{1}(v) ; h_{2}(v)\right)$, where $h_{j}(v)=D_{j} M^{-1}\left(\mu D^{T} v+\right.$ $u), j=1,2$. $M$ denotes the symmetric positive definite matrix which satisfies $M=\mu D^{T} D+I$.

Using the definition of $S$ and $h$, we can rewrite the three iterative steps in Algorithm 1 into

$$
\begin{aligned}
& u^{(m+1)}=\frac{w^{(m)}-\gamma+\sqrt{\left(w^{(m)}-\gamma\right)^{2}+4 \gamma u_{0}}}{2}, \\
& v^{(m+1)}=S\left(D_{1} w^{(m)} ; D_{2} w^{(m)}\right)=S \cdot h\left(v^{(m)}\right), \\
& w^{(m+1)}=M^{-1}\left(\mu D^{T} v^{(m+1)}+u^{(m+1)}\right),
\end{aligned}
$$

then the convergence results of Algorithm 1 can be proved as discussed in [34]. Firstly, as proved by Proposition 3.1 in [34], the nonexpansiveness of the shrinkage operator $S$ can be ensured. Then, it is easy to check that $M$ is nonsingularity, thus another symmetric positive definite matrix $T=D M^{-1} D^{T}$ is well defined and the spectral radius $\rho(T)<1$. Thus the operator $h$ is also nonexpansive. Secondly, the objective function in (25) is convex, bounded below, and coercive. Hence, for any fixed $\alpha_{1}>0$, the sequence $\left\{\left(v^{(m)}, w^{(m)}\right)\right\}$ generated by $(40),(41)$, and (42) from any star point $w^{(0)}$ converges to a solution of (25) (see [34, Theorem 3.4]). Furthermore, the convergence of $\left\{u^{(m)}\right\}$ to some $u^{*}$ follows directly from (40), hence we can conclude that for fixed $\alpha_{1}$ and $\alpha_{2}$, the sequence $\left\{\left(u^{(m)}, w^{(m)}\right)\right\}$ generated by Algorithm 1 from any start point $\left(u^{(0)}, w^{(0)}\right)$ converges to a fixed point $\left(u^{*}, w^{*}\right)$, which is the solution of (24) and (25).

4.6. Some Complexity Notes. It is clear that the complexity of the proposed algorithm mainly includes three parts. 
The calculation in (27) and (30) has a linear-time complexity of order $\mathrm{O}\left(N^{2}\right)$ for an $N$-by- $N$ image. Hence, the $u$ subproblem and $v$-subproblem can be solved quickly. The solution of the $w$-subproblem (32) requires three fast Fourier transforms and one inverse transform and a total complexity in the order of $\mathrm{O}\left(N^{2} \log \left(N^{2}\right)\right)=\mathrm{O}\left(N^{2} \log (N)\right)$.

\section{Numerical Results and Performance Analysis}

In this section, we demonstrate the effectiveness of our proposed Algorithm 1 in image denoising. The numerical results are compared with those obtained by the "HMW" method proposed by Huang et al. [21], "AA" method proposed by Aubert and Aujol [19], and the "RLO" method proposed by Rudin et al. [18]. In the tests, each pixel of an original image is degraded by a noise which follows a Gamma distribution with density function in (3) and $v$ being specified to have mean 1 and standard deviation $1 / \sqrt{L}$. The noise level is controlled by the value of $L$ in the experiments.

To compare the performance of the algorithms mentioned above, we compute the quality of restored images by the peak signal-to-noise ratio (PSNR), the improved SNR (ISNR), and the relative error (ReErr) of the restored image defined by

$$
\begin{aligned}
& \text { PSNR }=10 \log _{10}\left\{\frac{M N \max \{u\}^{2}}{\left\|u^{*}-u\right\|_{2}^{2}}\right\}, \\
& \text { ISNR }=10 \log _{10}\left\{\frac{\left\|u_{0}-u\right\|_{2}^{2}}{\left\|u^{*}-u\right\|_{2}^{2}}\right\}, \\
& \text { ReErr }=\frac{\left\|u_{0}-u\right\|_{2}^{2}}{\|u\|_{2}^{2}},
\end{aligned}
$$

where $u, u^{*}$, and $u_{0}$ are the original, the restored, and the observed images, respectively.

The solution of model (4) by the "RLO" method is obtained by using the following gradient projection iterative scheme [18]:

$$
\begin{aligned}
u^{(m+1)}=u^{(m)}+\Delta t & \operatorname{div}\left(\frac{\nabla u^{(m)}}{\sqrt{\left|\nabla u^{(m)}\right|^{2}+\varepsilon}}\right) \\
& \left.+\lambda \frac{u_{0}^{2}}{\left(u^{(m)}\right)^{3}}+\mu \frac{u_{0}}{\left(u^{(m)}\right)^{2}}\right] .
\end{aligned}
$$

Here, the two Lagrange multipliers $\lambda$ and $\mu$ are dynamically updated to satisfy the constraints (as explained in [18]).

The solution of the "AA" model (5) is obtained by using the following explicit iterative scheme [19]:

$$
u^{(m+1)}=u^{(m)}+\Delta t\left[\operatorname{div}\left(\frac{\nabla u^{(m)}}{\sqrt{\left|\nabla u^{(m)}\right|^{2}+\varepsilon}}\right)+\lambda \frac{u_{0}-u^{(m)}}{\left(u^{(m)}\right)^{2}}\right] .
$$

Here, the regularization parameter $\lambda$ is dynamically updated (as explained in [7]). In the "RLO" and "AA" methods, $\varepsilon$ is set to be $10^{-4}$, and $\Delta t$ is set to be a small positive number to ensure the convergence of the iterative scheme.

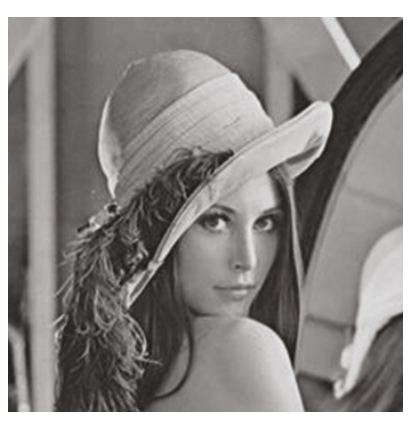

(a)

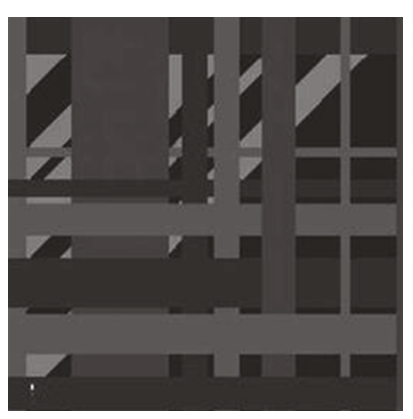

(b)

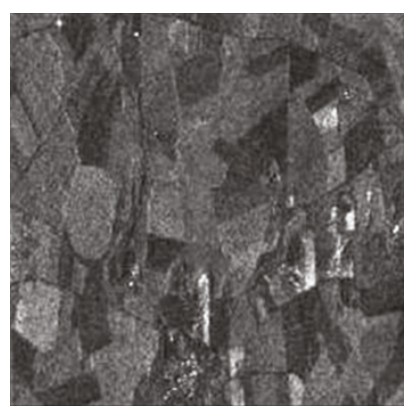

(c)

Figure 1: (a) The original "Lena" image; (b) the synthetic image "SynImag"; (c) the SAR image.

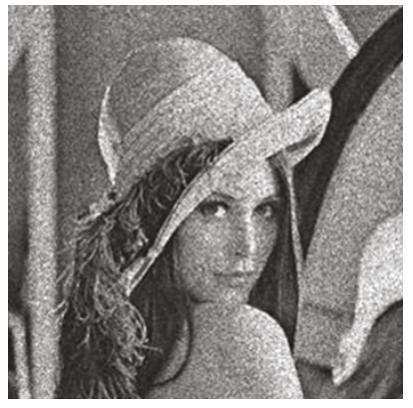

(a)

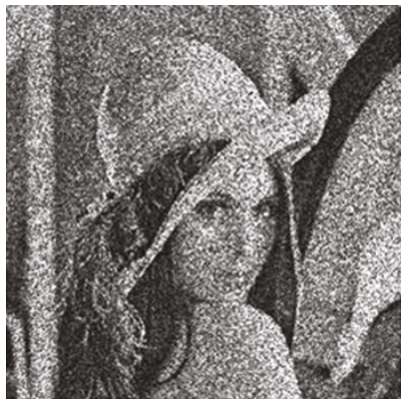

(b)
Figure 2: The degraded "Lena" image with $L=33$ (a) and the degraded "Lena" image with $L=5$ (b).

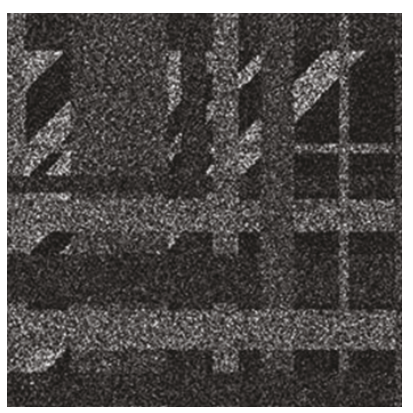

(a)

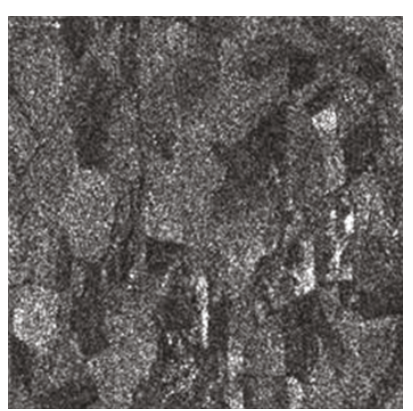

(b)
FIgure 3: The degraded "SynImag" image with $L=2$ (a) and the degraded SAR image with $L=10$ (b). 


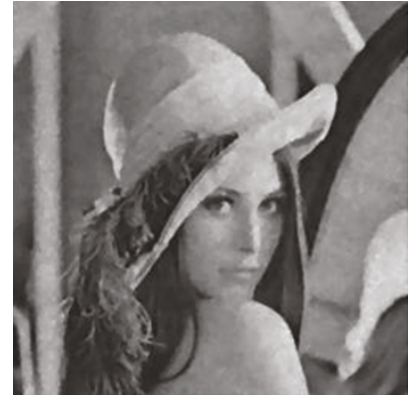

(a)

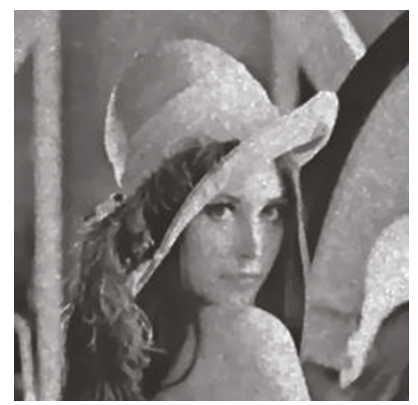

(c)

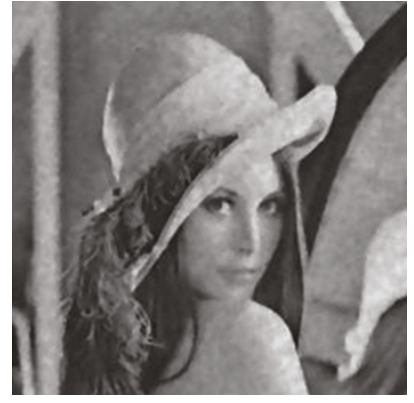

(b)

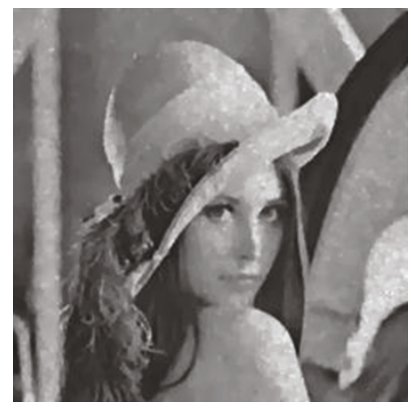

(d)

Figure 4: The restored "Lena" images for denoising Figure 2(a): (a) by the proposed method $\left(\alpha_{1}=0.9 \times 2^{32}, \alpha_{2}=1.5 \times 2^{32}\right.$, and $\zeta=$ $\left.5 \times 10^{-3}\right)$; (b) by the "HMW" method $\left(\alpha_{1}=0.01, \alpha_{2}=0.004\right)$; (c) by the "AA" method; (d) by the "RLO" method.

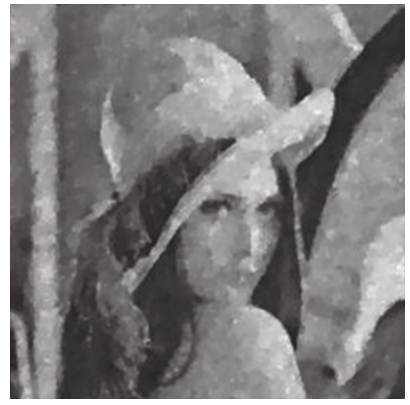

(a)

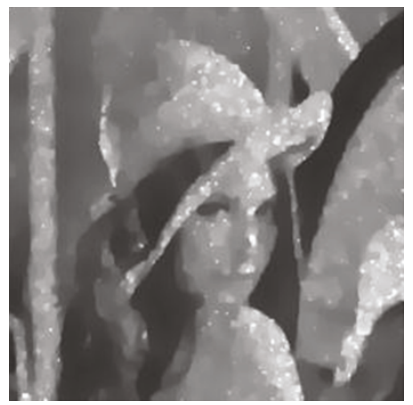

(c)

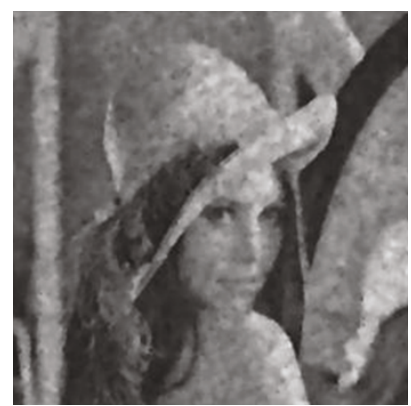

(b)

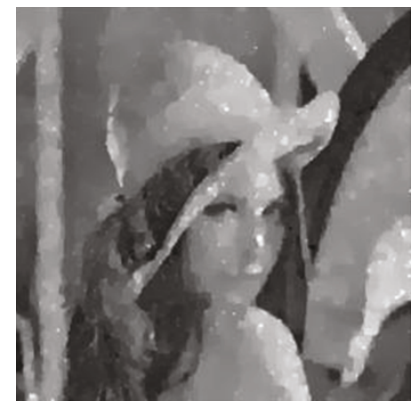

(d)

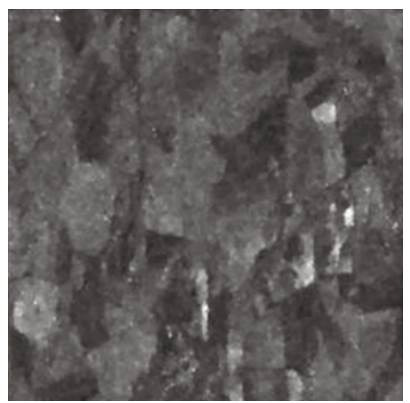

(a)

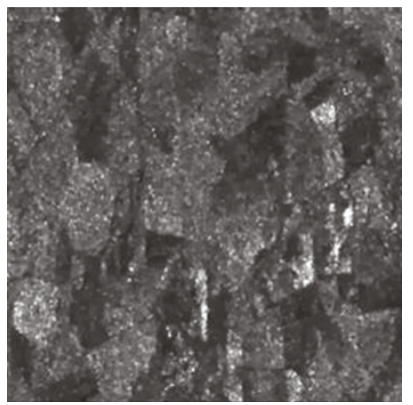

(c)

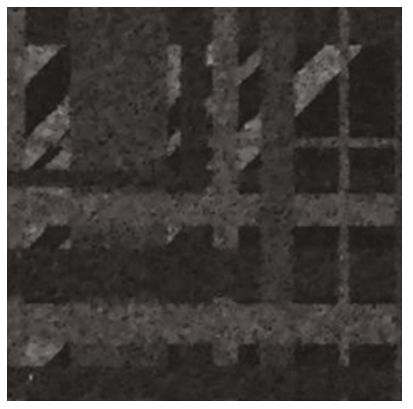

(b)

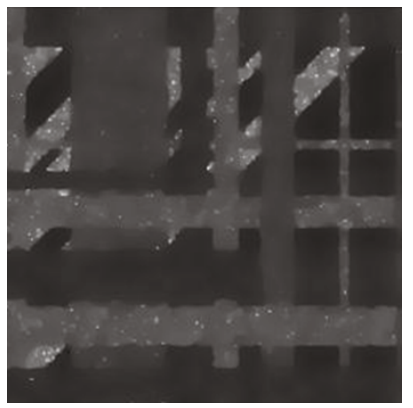

(d)

FIGURE 6: The restored "SynImag" images for denoising of Figure 3(a): (a) by the proposed method $\left(\alpha_{1}=4.6 \times 2^{32}, \alpha_{2}=\right.$ $1.5 \times 2^{32}$, and $\left.\zeta=5 \times 10^{-3}\right)$; (b) by the "HMW" method $\left(\alpha_{1}=\right.$ $0.01, \alpha_{2}=0.01$ ); (c) by the "AA" method; (d) by the "RLO" method.

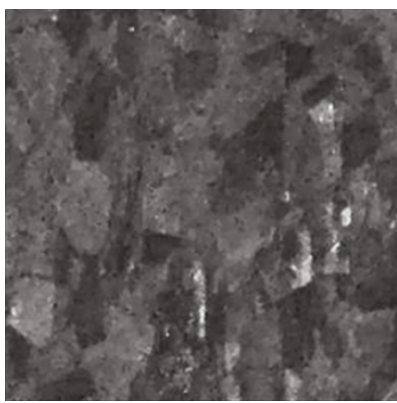

(b)

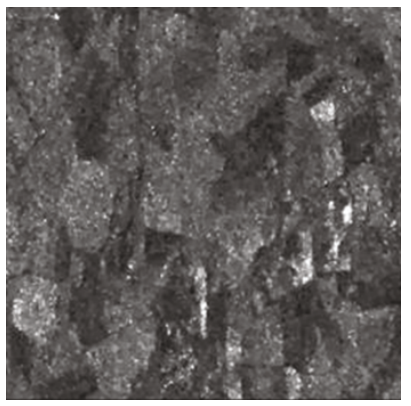

(d)

FIGURE 5: The restored "Lena" images for denoising Figure 2(b): (a) by the proposed method $\left(\alpha_{1}=0.5 \times 2^{32}, \alpha_{2}=0.2 \times 2^{32}\right.$, and $\zeta=$ $\left.5 \times 10^{-3}\right)$; (b) by the "HMW" method ( $\left.\alpha_{1}=0.01, \alpha_{2}=0.01\right)$; (c) by the "AA" method; (d) by the "RLO" method.

FIGURE 7: The restored SAR images for denoising of Figure 3(b): (a) by the proposed method $\left(\alpha_{1}=4 \times 2^{32}, \alpha_{2}=18 \times 2^{32}\right.$, and $\zeta=$ $\left.5 \times 10^{-3}\right)$; (b) by the "HMW" method ( $\left.\alpha_{1}=0.01, \alpha_{2}=0.004\right)$; (c) by the "AA" method; (d) by the "RLO" method. 


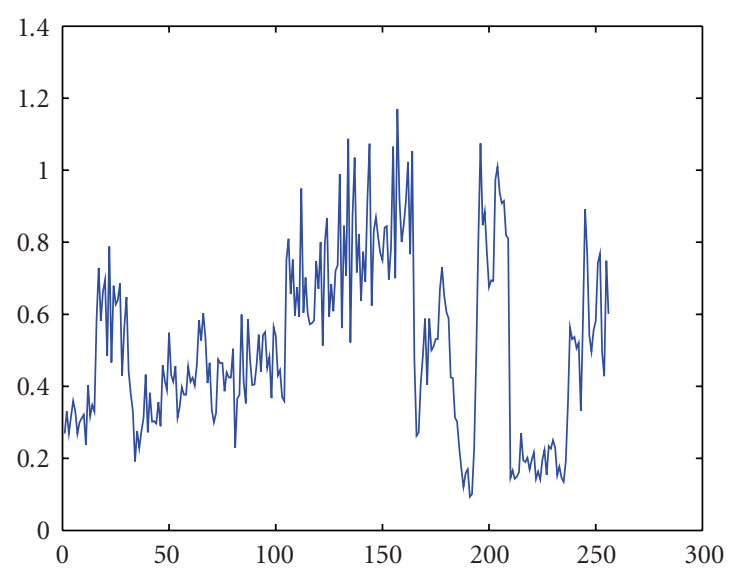

(a)

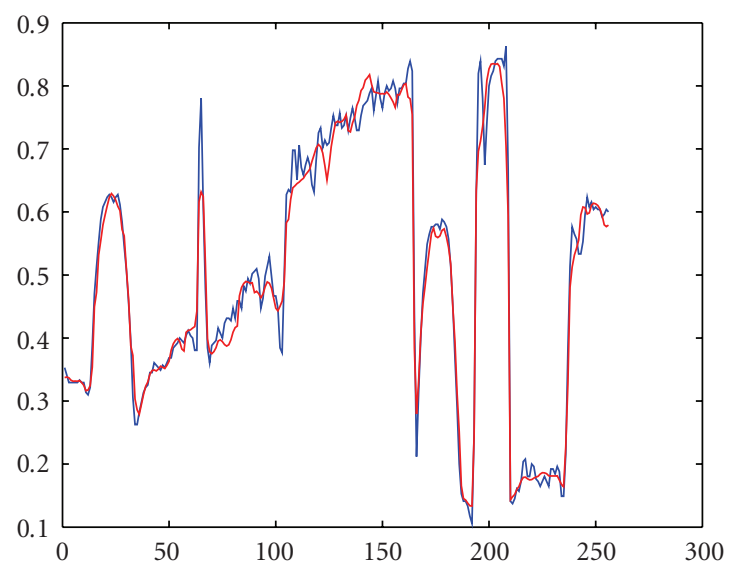

(c)

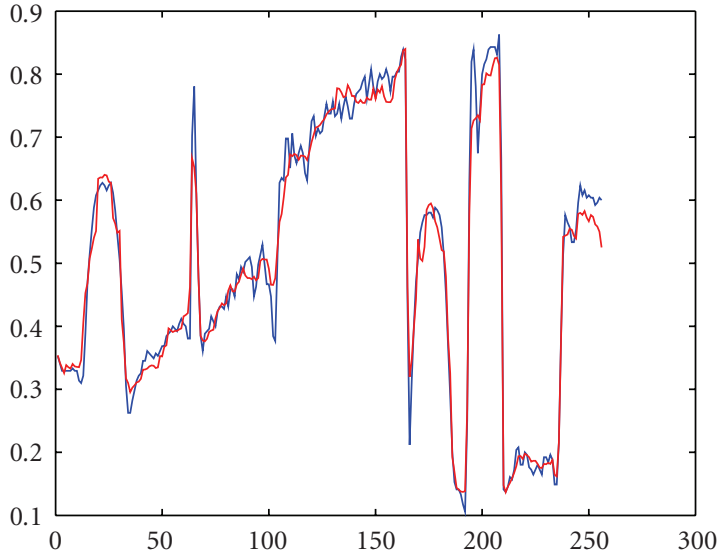

(b)

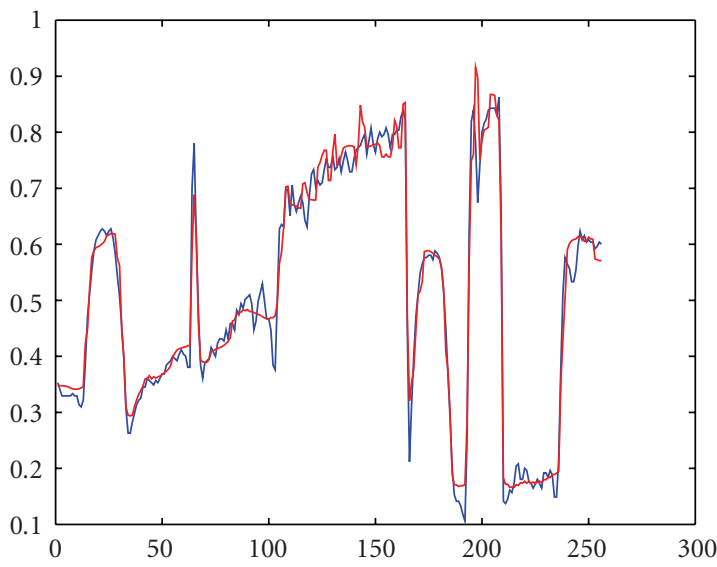

(d)

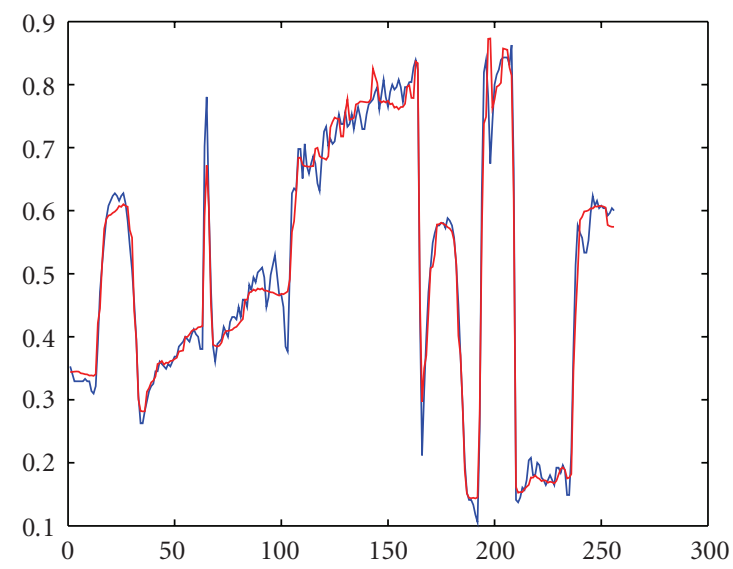

(e)

Figure 8: The 60th line of the original, noisy, and restored images for denoising Figure 2(a). (a) The noisy image; (b) the slice restored by the proposed method; (c) the slice restored by the "HMW" method; (d) the slice restored by the "AA" method; (e) the slice restored by the "RLO" method. Here the blue line is the original image, and the red line is the restored image.

The solution of the "HMW" model [21]

$$
\min _{z, w}\left\{\sum_{i=1}^{N^{2}}\left([z]_{i}+\left[u_{0}\right]_{i} e^{-[z]_{i}}\right)+\alpha_{1}\|z-w\|_{2}^{2}+\alpha_{2} \operatorname{TV}(w)\right\}
$$

is obtained by using the following alternating minimization algorithm:

$$
z^{(m)}=\arg \min _{z} \sum_{i=1}^{N^{2}}\left([z]_{i}+\left[u_{0}\right]_{i} e^{-[z]_{i}}\right)+\alpha_{1}\left\|z-w^{(m-1)}\right\|_{2}^{2},
$$




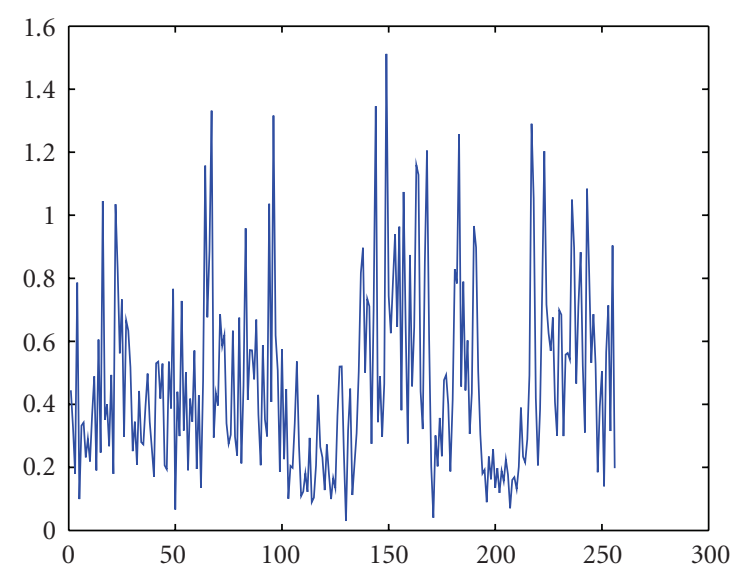

(a)

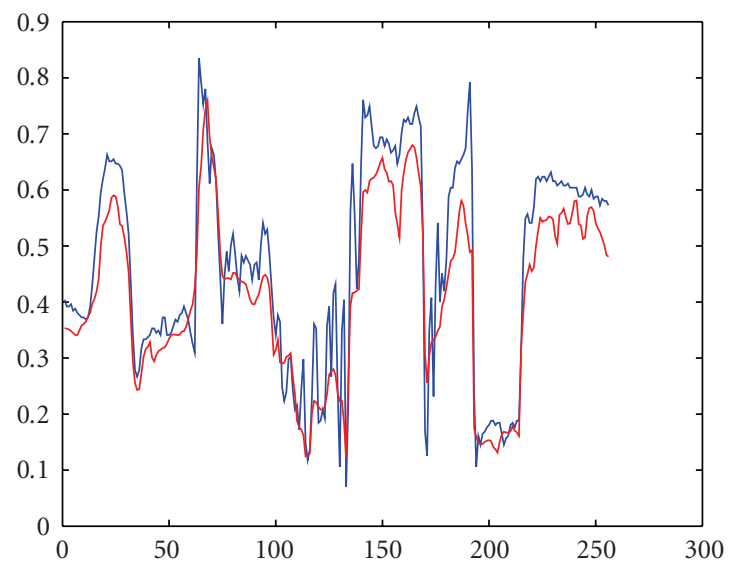

(c)

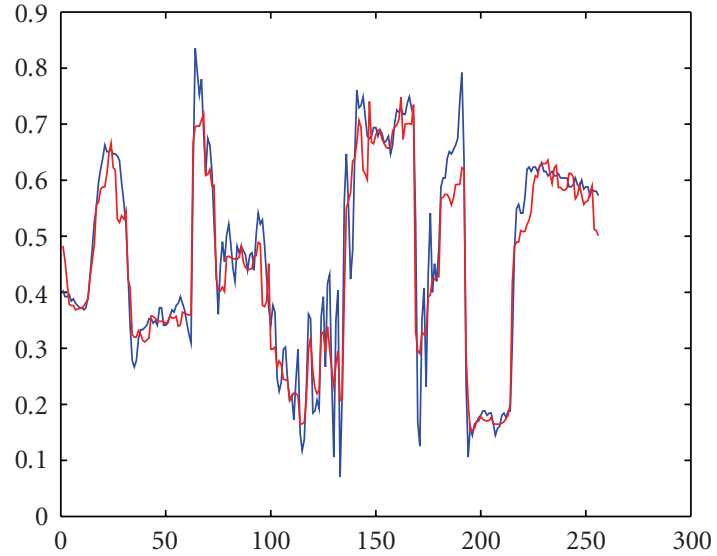

(b)

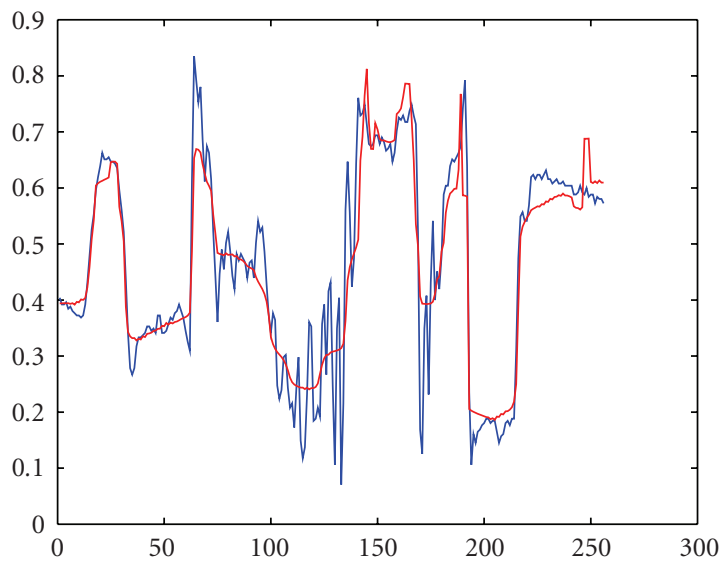

(d)

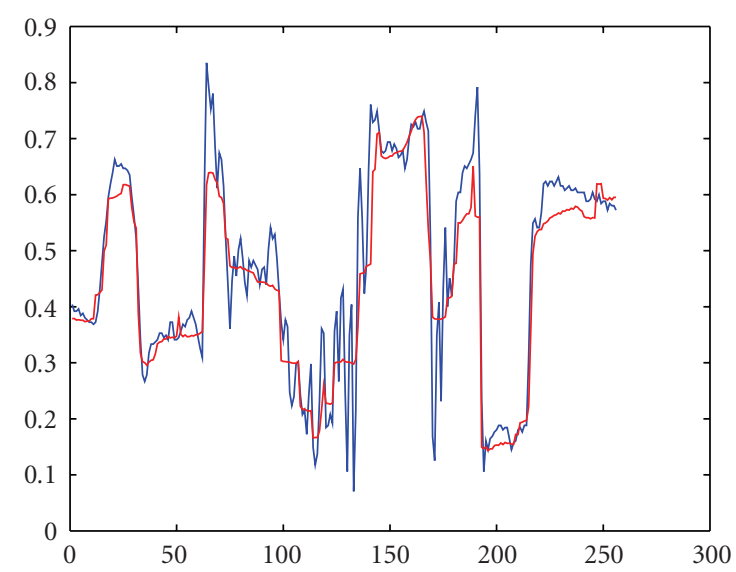

(e)

Figure 9: The 100th line of the original, noisy, and restored images for denoising Figure 2(b). (a) The noisy image; (b) the slice restored by the proposed method; (c) the slice restored by the "HMW" method; (d) the slice restored by the "AA" method; (e) the slice restored by the "RLO" method. Here the blue line is the original image, and the red line is the restored image.

$$
\begin{array}{r}
1-\left[u_{0}\right]_{i} e^{-[z]_{i}}+2 \alpha_{1}\left([z]_{i}-\left[w^{(m-1)}\right]_{i}\right)=0, \\
i=1,2, \ldots, N^{2},
\end{array}
$$

$$
w^{(m)}=\arg \min _{w}\left\{\alpha_{1}\left\|z^{(m)}-w\right\|_{2}^{2}+\operatorname{TV}(w)\right\} .
$$

The corresponding nonlinear Euler-Lagrange equation of subproblem (47) was solved using the Newton method. The Chambolle projection algorithm was employed in the denoising subproblem (47), and then the restored image was computed by $\exp (w)$. 


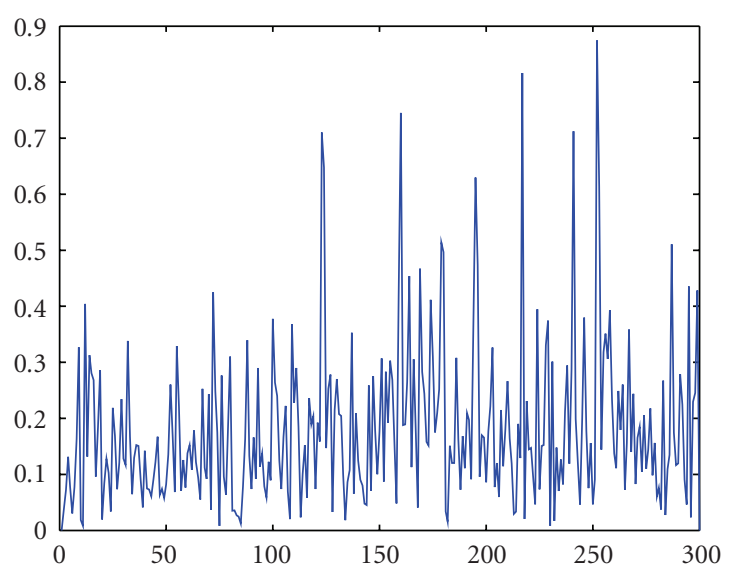

(a)

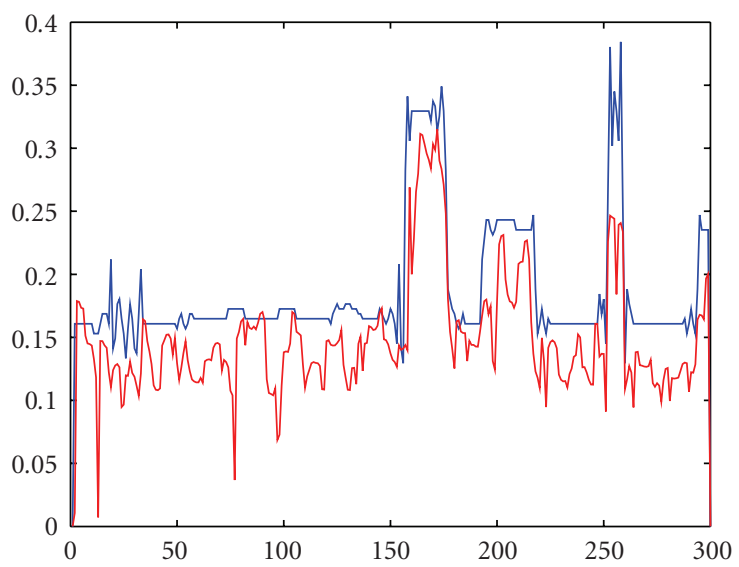

(c)

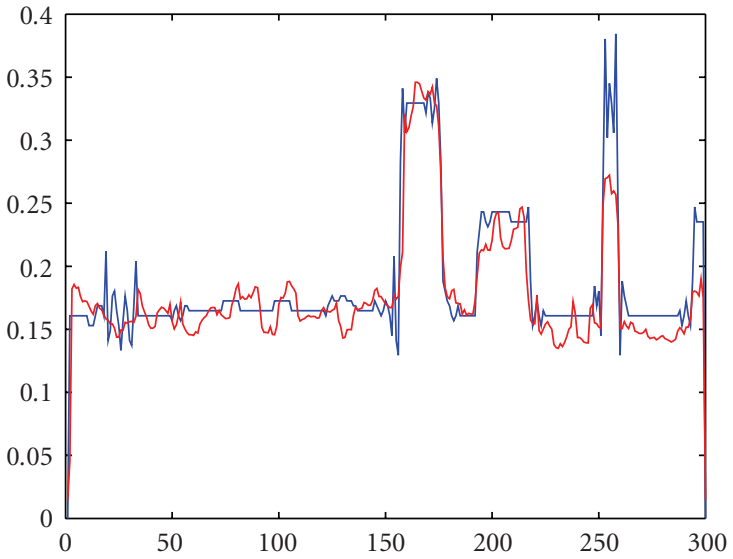

(b)

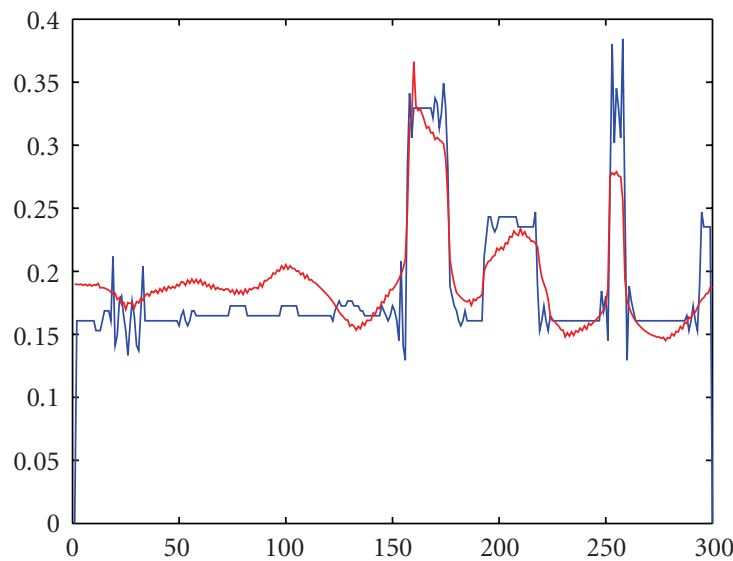

(d)

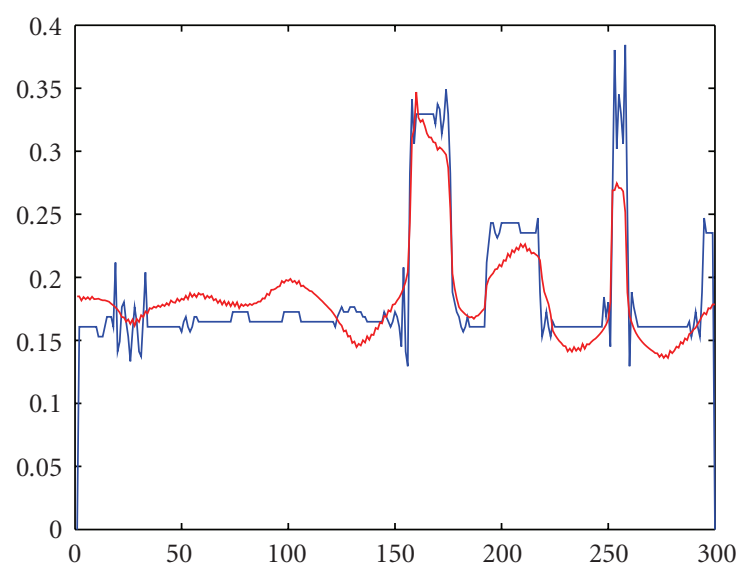

(e)

FIGURE 10: The 128th line of the original, noisy, and restored images for denoising Figure 3(a). (a) The noisy image; (b) the slice restored by the proposed method; (c) the slice restored by the "HMW" method; (d) the slice restored by the "AA" method; (e) the slice restored by the "RLO" method. Here the blue line is the original image, and the red line is the restored image.

The stopping criterion of the "HMW" method, the "AA" method, and the "RLO" method is that the PSNR value of the restored image reaches its maximum.

Our numerical experiments use several natural and manmade images such as "Lena" (Figure 1(a)), the synthetic image (Figure 1(b)), and the SAR image (Figure 1(c)) to compare various algorithms' performances. As shown in Figure 2, the "Lena" images are corrupted by a Gamma noise with $L=33$ (Figure 2(a)) and $L=5$ (Figure 2(b)). Figure 3(a) shows the noisy synthetic image which is 


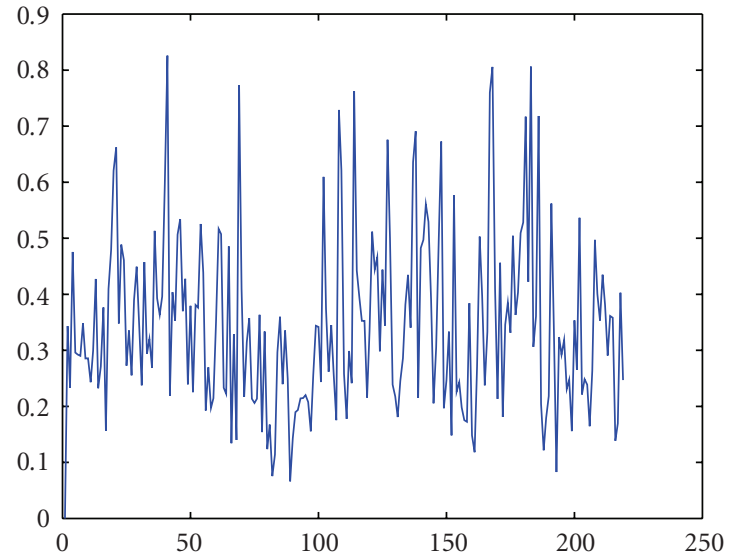

(a)

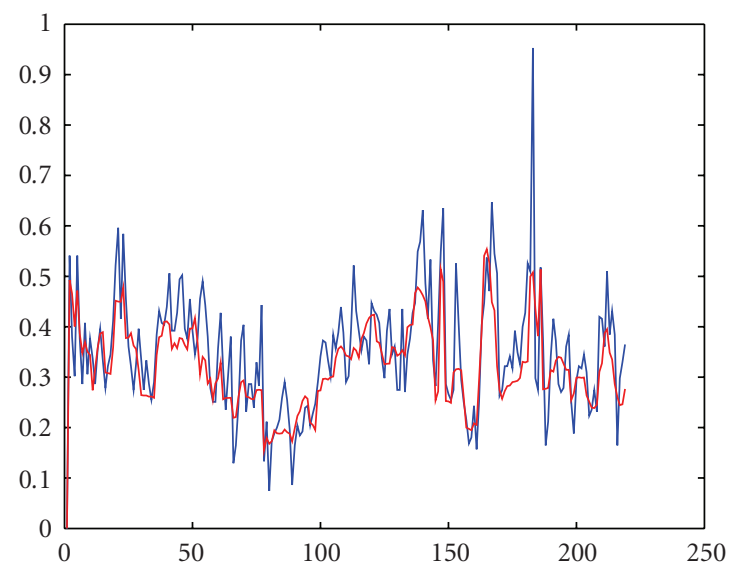

(c)

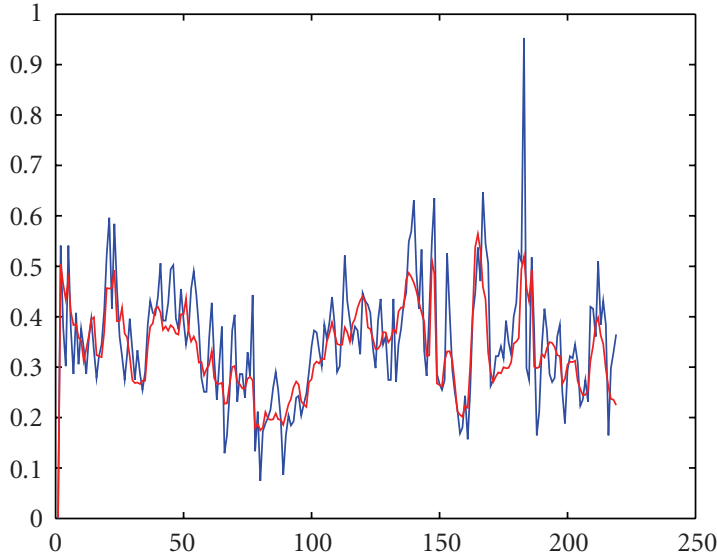

(b)

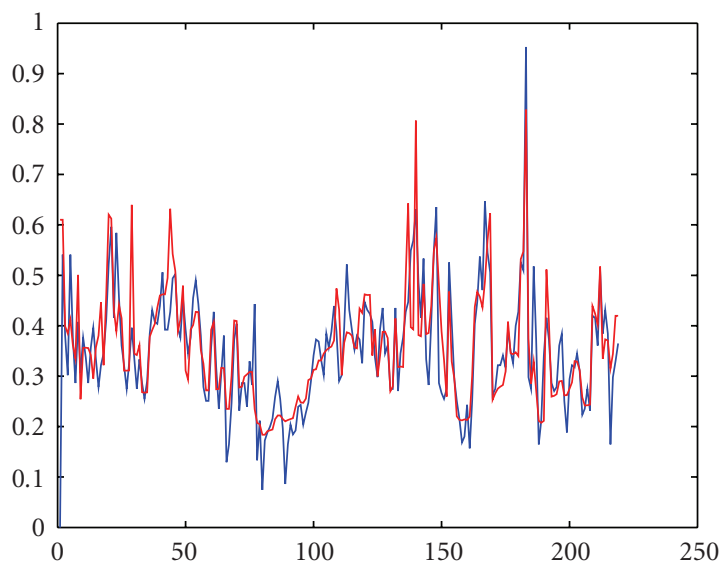

(d)

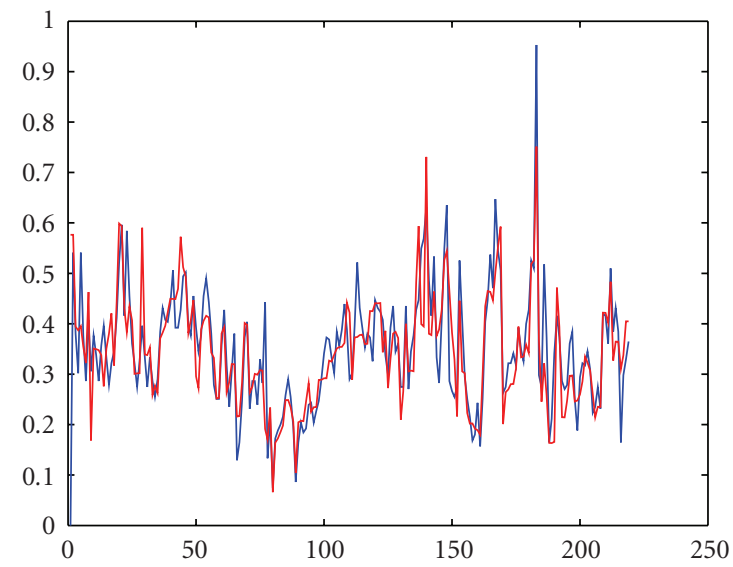

(e)

Figure 11: The 128th line of the original, noisy, and restored images for denoising Figure 3(b). (a) The noisy image; (b) the slice restored by the proposed method; (c) the slice restored by the "HMW" method; (d) the slice restored by the "AA" method; (e) the slice restored by the "RLO" method. Here the blue line is the original image, and the red line is the restored image.

corrupted by a Gamma noise with $L=2$, while Figure 3(b) shows the noisy SAR image which is distorted by Gamma noise with $L=10$.

Figures 4, 5, 6, and 7 show the restored "Lena," synthetic image and SAR images, respectively. In these experiments, the initial guess is set to be $u^{(0)}=u_{0}$. It is clear that the restoration results by the proposed method are visually much better than those by the "HMW" method, the "AA" method and the "RLO" method, especially when the noise variance is large, that is, when $L$ is small. The effectiveness of our 
TABLE 1: The PSNR, ISNR, and ReErr of the restored images using four methods.

\begin{tabular}{lcccccccccccc}
\hline \multirow{2}{*}{ Experiments } & \multicolumn{3}{c}{ Our algorithm } & \multicolumn{3}{c}{ "HMW" algorithm } & \multicolumn{3}{c}{ "AA" algorithm } & \multicolumn{3}{c}{ "RLO" algorithm } \\
& PSNR & ISNR & ReErr & PSNR & ISNR & ReErr & PSNR & ISNR & ReErr & PSNR & ISNR & ReErr \\
\hline "Lena" in Figure 2(a) & 27.973 & 7.914 & 0.0049 & 27.439 & 7.419 & 0.0056 & 27.016 & 6.950 & 0.0061 & 27.646 & 7.580 & 0.0053 \\
"Lena" in Figure 2(b) & 23.457 & 11.573 & 0.0139 & 21.032 & 9.131 & 0.0244 & 22.554 & 10.702 & 0.0171 & 23.143 & 11.291 & 0.0150 \\
"SynImag" in Figure 3(a) & 26.961 & 12.535 & 0.0279 & 22.338 & 7.860 & 0.0808 & 25.717 & 11.292 & 0.0371 & 25.841 & 11.416 & 0.0361 \\
"SynImag" in Figure 3(b) & 25.031 & 5.950 & 0.0252 & 24.579 & 5.589 & 0.0279 & 22.451 & 3.370 & 0.0456 & 22.657 & 3.5770 & 0.0435 \\
\hline
\end{tabular}

TABLE 2: The number of iterations and computational times of four algorithms.

\begin{tabular}{lcccccccc}
\hline \multirow{2}{*}{ Experiments } & \multicolumn{2}{c}{ Our algorithm } & \multicolumn{2}{c}{ "HMW" algorithm } & \multicolumn{2}{c}{ "AA" algorithm } & \multicolumn{2}{c}{ "RLO" algorithm } \\
& CPU time (s) & $\begin{array}{c}\text { Iterative } \\
\text { no. }\end{array}$ & CPU time (s) & $\begin{array}{c}\text { Iterative } \\
\text { no. }\end{array}$ & CPU time (s) & $\begin{array}{c}\text { Iterative } \\
\text { no. }\end{array}$ & CPU time (s) & $\begin{array}{c}\text { Iterative } \\
\text { no. }\end{array}$ \\
\hline "Lena" in Figure 2(a) & 8.438 & 77 & 8.609 & 72 & 39.860 & 278 & 51.938 & 115 \\
"Lena" in Figure 2(b) & 10.594 & 103 & 9.438 & 92 & 33.984 & 274 & 125.13 & 280 \\
"SynImag" in Figure 3(a) & 16.188 & 111 & 24.078 & 275 & 43.688 & 252 & 158.88 & 260 \\
"SAR" image in Figure 3(b) & 7.578 & 69 & 7.469 & 110 & 38.938 & 272 & 20.563 & 67 \\
\hline
\end{tabular}

proposed method is confirmed by the computing results of PSNR, ISNR, and ReErr of the three methods, as listed in Table 1. From Table 1, we can see that the PSNRs and ISNRs of the images restored by using our method are more than those restored by using the other three methods, and the relative errors ReErr are less than the other three methods. Moreover, our proposed algorithm is efficient. Table 2 shows the number of iterations required for convergence and the computational times required by our method, the "HMW" method, the "AA" method, and the "RLO" method. Although our method takes little more time than the "HMW" method in some experiments, it is clear that the computational time required by our method is quite competitive with the other two methods.

We check the homogeneity of regions of interest in the image and analyze the loss (or the preservation) of contrast. In Figures 8, 9, 10, and 11, we show several lines of the original, noisy, and restored images. It is clear from the figures that the lines restored by the proposed method are better than those restored by the other three methods. Table 3 lists the results of mean square errors (MSEs) between the corresponding line of the original images and one of the restored images in Figures 8-11. From the computing MSE results, we can conclude that our algorithm has the best performance.

We also prove that the proposed algorithm does not depend on starting point by experiment. In our experiment, we use two different initial guessed images in the proposed method. The first initial guess is set to be $u^{(0)}=u_{0}$, and the second is set to be a constant image where the constant is the mean of $u_{0}$. Here we do not give the restored images using the mean image as initial guess, because they are almost the same with those using the $u^{(0)}=u_{0}$. Table 4 shows that the PSNRs, ISNRs, and relative errors of the restored images using two different initial guesses are about the same. These means that our method is not sensitive to initial guess.

\section{Conclusion}

In this paper, we have investigated a new nonconvex variational model for the multiplicative noise removal problem under the Weberized TV regularization framework. Using a reference energy functional, we propose another surrogate strictly convex objective function for multiplicative noise removal. Our algorithm is designed using the well-known variable-splitting and penalty techniques in optimization. The proposed algorithm contains three subminimizing parts and each of them permits a closed-form solution. Our experimental results show that our proposed algorithm is effective and efficient to filter out multiplicative noise while well preserving the significant details.

\section{Appendix}

\section{Proof of Theorem 1}

Let us denote that $\alpha=\inf _{\Omega}\left(u_{0}\right), \beta=\sup _{\Omega}\left(u_{0}\right), \operatorname{TV}(u)=$ $\int_{\Omega}|\nabla u| \mathrm{d} x, E_{\mathrm{data}}(u)=\int_{\Omega}\left(u-u_{0} \log u\right) \mathrm{d} x$, and $h(s)=s-$ $u_{0} \log s$. It is obvious that $h^{\prime \prime}(s)=u_{0} / s^{2}$ and $h$ is strictly convex as $u_{0}>0$. Therefore, we have $h(s) \geq u_{0}-u_{0} \log u_{0}$ for all $s \in R^{2}$. This implies that $E_{R}(u)$ has a lower bound for all $u \in \operatorname{BV}(\Omega)$. Hence we consider a minimizing sequence $\left\{u_{n}\right\} \in \mathrm{BV}(\Omega)$ for $(16)$.

First, we show that $\alpha \leq u_{n} \leq \beta$. Since $u_{0} \in L^{\infty}(\Omega)$ is a positive, bounded function with $\inf _{\Omega} u_{0}>0$, we choose a sequence $\left\{u_{n}\right\} \in C^{\infty}(\bar{\Omega})$ such that $u_{n} \rightarrow u_{0}$ in $L^{1}(\Omega)$ and a.e. in $\Omega$ as $n \rightarrow \infty$, and $\inf _{\Omega}\left(u_{0}\right) \leq u_{n} \leq \sup _{\Omega}\left(u_{0}\right)$. We remark that $h(s)$ is decreasing as $s \in\left(-\infty, u_{0}\right)$ and increasing as $s \in\left(u_{0},+\infty\right)$. Therefore, if $M>u_{0}$, one always has $h(\min (s, M)) \leq h(s)$. Hence, if we let $M=\beta=\sup \left(u_{0}\right)$, then

$$
E_{\text {data }}(\inf (u, \beta)) \leq E_{\text {data }}(u) .
$$


TABLE 3: The mean square error between the line of the original images and the restored images using four algorithms.

\begin{tabular}{|c|c|c|c|c|}
\hline Experiments & Our algorithm & "HMW" algorithm & "AA" algorithm & "RLO" algorithm \\
\hline "Lena" in Figure 2(a) & 0.4892 & 0.5134 & 0.5512 & 0.5034 \\
\hline "Lena" in Figure 2(b) & 0.8905 & 1.2748 & 1.0664 & 1.0778 \\
\hline "SynImag" in Figure 3(a) & 0.4147 & 0.5309 & 0.5502 & 0.5355 \\
\hline "SAR" image in Figure 3(b) & 1.0903 & 1.1204 & 1.1729 & 1.1294 \\
\hline
\end{tabular}

TABLE 4: The PSNR, ISNR, and ReErr of the restored images using two different initial guesses in our algorithms.

\begin{tabular}{|c|c|c|c|c|c|c|}
\hline \multirow{2}{*}{ Experiments } & \multicolumn{3}{|c|}{$u^{(0)}=u_{0}$} & \multicolumn{3}{|c|}{$u^{(0)}=\operatorname{mean}\left(u_{0}\right)$} \\
\hline & PSNR & ISNR & ReErr & PSNR & ISNR & ReErr \\
\hline "Lena" in Figure 2(a) & 27.973 & 7.914 & 0.0049 & 28.001 & 7.981 & 0.0049 \\
\hline "Lena" in Figure 2(b) & 23.457 & 11.573 & 0.0139 & 23.564 & 11.663 & 0.0136 \\
\hline "SynImag" in Figure 3(a) & 26.961 & 12.535 & 0.0279 & 27.003 & 12.524 & 0.0276 \\
\hline "SAR" image in Figure 3(b) & 25.031 & 5.950 & 0.0252 & 25.003 & 6.013 & 0.0253 \\
\hline
\end{tabular}

Moreover, we have that (e.g., see [37, Section 4.3, Lemma 1]):

$$
\operatorname{TV}(\inf (u, \beta)) \leq \operatorname{TV}(u) .
$$

Combining (A.1) and (A.2), we thus deduce that

$$
E_{R}(\inf (u, \beta)) \leq E_{R}(u) .
$$

And we get in the same way that

$$
E_{R}(\sup (u, \alpha)) \leq E_{R}(u) .
$$

Therefore, we can assume without restriction that $\inf _{\Omega}\left(u_{0}\right) \leq$ $u_{n} \leq \sup _{\Omega}\left(u_{0}\right)$.

Second, the above proof implies in particular that $u_{n}$ is bounded in $L^{1}(\Omega)$. Moreover, by the definition of $\left\{u_{n}\right\}$, we get that there exists a constant $C$ such that

$$
\operatorname{TV}\left(u_{n}\right)+\lambda E_{\text {data }}\left(u_{n}\right) \leq C .
$$

Moreover, standard computations show that $E_{\text {data }}\left(u_{n}\right)$ reaches its minimum value when $u=u_{0}$ and thus we deduce that $\operatorname{TV}\left(u_{n}\right) \leq C$.

Therefore, we get that $u_{n}$ is bounded in $\operatorname{BV}(\Omega)$ and there exists $u \in \mathrm{BV}(\Omega)$ such that up to a subsequence, $u_{n} \rightarrow u$ in $\mathrm{BV}(\Omega)$-weak* and $u_{n} \rightarrow u$ in $L^{1}(\Omega)$-strong. Necessarily, we have $0 \leq \alpha \leq u \leq \beta$, and thanks to the lower semicontinuity of the total variation and Fatou's lemma, we get that $u$ is a solution of problem (16).

Since $h$ is strictly convex as $u_{0}>0$, the uniqueness of the minimizer follows from the strict convexity of the energy functional in (16). This completes the proof.

\section{Acknowledgments}

The authors would like to express their gratitude to the anonymous referees for making helpful and constructive suggestions, especially for the proof of the algorithm's convergence. This work is supported in part by the Natural Science Foundation of China under Grant no. 60802039 and 60672074 and by the National 863 High Technology Development Project under Grant no. 2007AA12Z142.

\section{References}

[1] G. Steidl and T. Teuber, "Removing multiplicative noise by Douglas-rachford splitting methods," Journal of Mathematical Imaging and Vision, vol. 36, no. 2, pp. 168-184, 2010.

[2] T. Chan and J. Shen, Image Processing and Analysis-Variational, PDE, Wavelet, and Stochastic Methods, SIAM, Philadelphia, Pa, USA, 2005.

[3] G. Aubert and P. Kornprobst, Mathematical Problems in Image Processing, vol. 147 of Applied Mathematical Sciences, Springer, London, UK, 2002.

[4] M. A. T. Figueiredo and R. D. Nowak, "An EM algorithm for wavelet-based image restoration," IEEE Transactions on Image Processing, vol. 12, no. 8, pp. 906-916, 2003.

[5] J. M. Bioucas-Dias, "Bayesian wavelet-based image deconvolution: a GEM algorithm exploiting a class of heavy-tailed priors," IEEE Transactions on Image Processing, vol. 15, no. 4, pp. 937-951, 2006.

[6] J. Ma and G. Plonka, "Combined curvelet shrinkage and nonlinear anisotropic diffusion," IEEE Transactions on Image Processing, vol. 16, no. 9, pp. 2198-2206, 2007.

[7] L. I. Rudin, S. Osher, and E. Fatemi, "Nonlinear total variation based noise removal algorithms," Physica D, vol. 60, no. 1-4, pp. 259-268, 1992.

[8] J. P. Oliveira, J. M. Bioucas-Dias, and M. A. T. Figueiredo, "Adaptive total variation image deblurring: a majorizationminimization approach," Signal Processing, vol. 89, no. 9, pp. 1683-1693, 2009.

[9] A. Buades, B. Coll, and J. M. Morel, "A review of image denoising algorithms, with a new one," SIAM Journal on Multiscale Modeling and Simulation, vol. 4, no. 2, pp. 490-530, 2005.

[10] L. Alvarez, F. Guichard, P.-L. Lions, and J.-M. Morel, "Axioms and fundermental equations of image processing," Archive for Rational Mechanics and Analysis, vol. 16, no. 9, pp. 200-257, 1993.

[11] W.-Z. Shao and Z.-H. Wei, "Edge-and-corner preserving regularization for image interpolation and reconstruction," Image and Vision Computing, vol. 26, no. 12, pp. 1591-1606, 2008.

[12] H. Lewis, Principle and Applications of Imaging Radar, vol. 2 of Manual of Remote Sensing, John Wiley \& Sons, New York, NY, USA, 3rd edition, 1998. 
[13] J. S. Lee, "Digital enhancement and noise filtering by use of local statistics," IEEE Transactions on Pattern Analysis and Machine Intelligence, vol. 2, no. 2, pp. 165-168, 1980.

[14] D. T. Kuan, A. A. Sawchuk, T. C. Strand, et al., "Adaptive noise smoothing filter for images with signal dependent noise," IEEE Transactions on Pattern Analysis and Machine Intelligence, vol. 7, no. 2, pp. 165-177, 1985.

[15] Y. Yu and S. T. Acton, "Speckle reducing anisotropic diffusion," IEEE Transactions on Image Processing, vol. 11, no. 11, pp. 1260-1270, 2002.

[16] K. Krissian, C.-F. Westin, R. Kikinis, and K. G. Vosburgh, "Oriented speckle reducing anisotropic diffusion," IEEE Transactions on Image Processing, vol. 16, no. 5, pp. 1412-1424, 2007.

[17] G. J. Liu, X. P. Zeng, F. C. Tian, Z. Z. Li, and K. Chaibou, "Speckle reduction by adaptive window anisotropic diffusion," Signal Processing, vol. 89, no. 11, pp. 2233-2243, 2009.

[18] L. I. Rudin, P. L. Lions, and S. Osher, "Multiplicative denoising and deblurring: theory and algorithms," in Geometric Level Set Methods in Imaging, Vision, and Graphics, S. Osher and N. Paragios, Eds., pp. 103-120, 2003.

[19] G. Aubert and J. F. Aujol, "A variational approach to remove multiplicative noise," SIAM Journal on Applied Mathematics, vol. 68, no. 4, pp. 925-946, 2008.

[20] J. Shi and S. Osher, "A nonlinear inverse scale space method for a convex multiplicative noise model," SIAM Journal on Applied Mathematics, vol. 1, no. 3, pp. 294-321, 2008.

[21] Y. M. Huang, M. K. Ng, and Y. M. Wen, "New total variation method for multiplicative noise removal," SIAM Journal on Imaging Sciences, vol. 2, no. 1, pp. 22-40, 2009.

[22] S. Durand, J. Fadili, and M. Nikolova, "Multiplicative noise cleaning via a variational method involving curvelet coefficients," in Scale Space and Variational Methods, A. Lie, M. Lysaker, K. Morken, and X.-C. Tai, Eds., Springer, Voss, Norway, 2009.

[23] J. H. Shen, "On the foundations of vision modeling: I. Weber's law and Weberized TV restoration," Physica D, vol. 175, no. 3-4, pp. 241-251, 2003.

[24] J. H. Shen and Y.-M. Jung, "Weberized Mumford-Shah model with Bose-Einstein photon noise. ," Applied Mathematics and Optimization, vol. 53, no. 3, pp. 331-358, 2006.

[25] Z. H. Wei, Y. Q. Fu, Z. G. Gao, and S. X. Cheng, "Visual compander in wavelet-based image coding," IEEE Transactions on Consumer Electronics, vol. 44, no. 4, pp. 1261-1266, 1998.

[26] Z. H. Wei, P. Qin, Y. Q. Fu, and S. X. Cheng, "Perceptual digital watermark of images using wavelet transform," IEEE Transactions on Consumer Electronics, vol. 44, no. 4, pp. 12671272, 1998.

[27] E. H. Weber, "De pulsu, resorptione, audita et tactu," in Annotationes Anatomicae et Physiologicae, Koehler, Leipzig, Germany, 1834.

[28] L. C. Evans and R. F. Gariepy, Measure Thoery and Fine Properties of Functions, CRC Press, Boca Raton, Fla, USA, 1992.

[29] A. Chambolle, "An algorithm for total variation minimization and applications," Journal of Mathematical Imaging and Vision, vol. 20, no. 1-2, pp. 89-97, 2004.

[30] T. F. Chan, G. H. Golub, and P. Mulet, "A nonlinear primaldual method for total variation-based image restoration," SIAM Journal of Scientific Computing, vol. 20, no. 6, pp. 19641977, 1999.

[31] T. F. Chan and P. Mulet, "On the convergence of the lagged diffusivity fixed point method in total variation image restoration," SIAM Journal on Numerical Analysis, vol. 36, no. 2, pp. 354-367, 1999.
[32] T. F. Chan and J. H. Shen, Theory and computation of variational image deblurring, Lecture Notes Series, IMS, Singapore, 2006.

[33] T. F. Chan, J. H. Shen, and L. Vese, "Variational PDE models in image processing," Notices of the American Mathematical Society, vol. 50, pp. 14-26, 2003.

[34] Y. Wang, J. Yang, W. Yin, and Y. Zhang, "A new alternating minimization algorithm for total variation image reconstruction," SIAM Journal on Imaging Sciences, vol. 1, no. 3, pp. 248272, 2008.

[35] Y. Wang, J. Yang, W. Yin, and Y. Zhang, "A fast algorithm for image deblurring with total variation regularization," Tech. Rep. TR07-10, Rice University CAAM, Houston, Tex, USA.

[36] J. Nocedal and S. J. Wright, Numerical Optimization, Springer, Berlin, Germany, 2000.

[37] P. Kornprobst, R. Deriche, and G. Aubert, "Image sequence analysis via partial differential equations," Journal of Mathematical Imaging and Vision, vol. 11, no. 1, pp. 5-26, 1999. 